\title{
Doppler ultrasound and strain elastography in the assessment of cutaneous melanoma: preliminary results
}

\author{
Carolina Botar Jid', Sorana D. Bolboacă², Rodica Cosgarea ${ }^{3}$, Simona Şenilă ${ }^{3}$, Liliana Rogojan ${ }^{4}$, \\ Manuela Lenghel $^{1}$, Dan Vasilescu ${ }^{1}$, Sorin M. Dudea ${ }^{1}$
}

${ }^{1}$ Department of Radiology," Iuliu Haţieganu" University of Medicine and Pharmacy, ${ }^{2}$ Department of Medical Informatics and Biostatistics, "Iuliu Haţieganu" University of Medicine and Pharmacy, ${ }^{3}$ Department of Dermatology "Iuliu Haţieganu" University of Medicine and Pharmacy, ${ }^{4}$ Emergency County Hospital Cluj-Napoca, Department of Pathology, Cluj-Napoca, Romania

\begin{abstract}
Aim: To study the usefulness of color or power Doppler ultrasound (US) in the pre-surgical evaluation of skin melanoma, and to correlate the Doppler characteristics with the appearance on high frequency ultrasound strain elastography (SE) in the preoperative evaluation of cutaneous melanoma. Materials and method: The study included 42 cutaneous melanoma lesions in 39 adult subjects examined between September 2011 and January 2015. Doppler US features (the presence and aspect of vascularization, and the number of vascular pedicles) and elasticity by strain elastography were evaluated together with the pathological results. Results: The melanoma lesions presented hyper-vascularization, with multiple vascular pedicles and stiff appearance. Significant correlations between the thickness of the tumor, measured histopathologically by the Breslow index, and the degree of vascularization $(p=0.0167)$, and number of vascular pedicles $(p=0.0065)$ were identified. Strong correlations between the SE appearance and vascularization on one hand, and SE and the number of vascular pedicles were also identified $(\mathrm{p}<0.001)$. Conclusion: Our study demonstrates that Doppler US and SE offer useful information for THE preoperative evaluation of cutaneous melanoma and may contribute to better defining the long term prognosis.
\end{abstract}

Keywords: cutaneous melanoma, Doppler ultrasound, strain elastography, real time elastography

\section{Introduction}

Cutaneous melanoma (CM) is the most important skin tumor with increasing incidence in the general population, including young people, with major health and socio-economic implications [1-3]. According to Scally et al, in 2013 there were about 76,250 new cases of CM which produced 9180 deaths [4] while according to $\mathrm{Li}$ et al about 76,100 new cases were diagnosed in 2014 in the USA with an estimated 9710 expected deaths [5]. Pa-

Received 08.08.2015 Accepted 24.09.2015

Med Ultrason

2015, Vol. 17, No 4, 509-514

Corresponding author: Sorana D. Bolboacă

Department of Medical Informatics

and Biostatistics, "Iuliu Haţieganu"

University of Medicine and Pharmacy

6, Louis Pasteur street

400349 Cluj-Napoca, Romania

E-mail: sbolboaca@gmail.com tients with CM present a high risk of relapsing, the occurrence of metastases, and a low rate of long-term survival [5]. The prognosis of these patients depends not only on the thickness of the tumor (Breslow index), presence of ulceration, mitotic rate, or Clark level invasion [6,7], but also on tumor vascularization, which is correlated with the risk of recurrence, occurrence of metastasis, and reduced long-term survival [8-12].

To establish a complex, highly accurate preoperative diagnosis in patients with CM is necessary for the best therapeutic assessment in order to improve the survival rate. Ultrasound (US) has an important role in diagnosis and staging and for establishing the optimal treatment and follow-up of patients with CM [10,11]. Using high frequency transducers, US allow a good characterization of the skin tumors and assessment of the thickness of the tumor (ultrasound Breslow index) [10-12]. By using Doppler techniques, US detect the presence of vascular signal, describe the distribution and characterize intra- 
tumoral vessels, thus increasing the diagnostic accuracy [10-13].

Strain elastography (SE) offers information about the relative elasticity or stiffness of the tissues comparing the target lesion with surrounding normal tissues, on the assumption that stiff tissues (as in most malignancies) are less deformable than the soft tissues (basically benign) $[14,15]$. The degree of elasticity is color coded, the elastographic information being superimposed on the twodimensional image.

The aim of this study was to assess the usefulness of color or power Doppler US in the pre-surgical evaluation of skin melanoma in relation with pathology results, and to correlate the information obtained by Doppler with the high frequency SE appearance in the preoperative evaluation of $\mathrm{CM}$.

\section{Material and methods}

A prospective study was performed between September 2011 and January 2015. The study comprised patients with skin lesions having the clinical suspicion of malignant melanoma, without previous cutaneous biopsy or surgery at the level of the identified lesion.

The exclusion criteria were: patients without specialized clinical examination, with other type of skin lesions than melanoma at clinical examination, patients who had underwent any dermatological treatment, and patients with previous biopsy or surgery in the area of the lesion.

All images were obtained using an Ultrasonix Sonotouch scanner (Ultrasonix Medical Corporation, Richmond, Canada) with an 8-40 MHz linear transducer and the possibility to perform Doppler ultrasound and SE.

The imaging parameters for Doppler ultrasound examinations were set to increase the detection of lowvelocity and low-volume flows (color Doppler: PRF 500-1000 Hz, wall filter 25-50 Hz, power Doppler: PRF $350-700 \mathrm{~Hz}$, wall filter 22-50 Hz; color and power Doppler: color gain maximized for optimal sensitivity while avoiding excessive color noise, color vs. echo priority ranging from 70 to $90 \%$ and color persistence adjusted to high values). The tumors were scanned in longitudinal and transverse planes. The ultrasound gel was in sufficient quantity so that the transducer exerted no compression, to avoid alteration of tumor thickness or blood flow.

For SE investigation each skin lesion was scanned in transverse and longitudinal planes with compression applied perpendicular to the lesion. A wide color window was used in order to include both the lesion and surrounding normal epidermis, dermal, and hypodermal tissue. Whenever possible, more than half of the region of interest width contained non-tumoral tissue.
Images with a good acquisition quality scale, defined as midrange quality scale light green coloring, were stored. Three different images of Doppler US (with the most representative vascular signal) and SE (considered by the examiner to be representative and reproducible for the lesion) were stored for each lesion, for further analysis.

The following information was collected for each patient:

- Doppler US: the aspect of vascularization (no or low vascularization - few color spots; medium vascularization - vascular signal $<50 \%$ of tumor; hypervascularization - vascular signal $>50 \%$ of the tumor) and the number of vascular pedicles (single or multiple);

- Qualitative SE: intratumoral distribution of colors (soft tissues were coded in red, medium elasticity tissues were colored in shades of yellow and green, and stiff tissues appeared in shades of blue). We used qualitative distribution of elasticity to correlate with the degree of vascularization: low elasticity for more than $50 \%$ of blue shades in the tumor; medium elasticity for predominant shades of yellow and green and high elasticity for more than $50 \%$ red color inside of the skin tumors. For Doppler examinations the images of the most visible vascular signal were analyzed. A single SE image, considered by the examiner to be the most representative and reproducible for the lesion, was also retained for analysis. All Doppler and SE examinations were performed by the same radiologist with 12 years experience of US and 8 years experience of SE.

Informed consent was obtained from each patient included in the study. The study protocol was approved by the Ethical Committee of the institutions.

\section{Statistical analysis}

Qualitative data were summarized as percentages and associated $95 \%$ confidence intervals (CI, provided in squared brackets), confidence intervals being calculated with an exact method [16]. The Breslow index was summarized as a mean and standard deviation since it proved to be normally distributed.

The comparison between the two proportions was made using the Z-test. The association in the contingency tables was carried out with the Fisher exact test. The relationship between the Doppler characteristics and the Breslow index and respectively between the SE appearance and the Doppler characteristics was investigated using the Spearman correlation coefficient.

Statistical analysis was conducted with Statistica software (v.8) at a significance level of 5\% for comparisons of two groups and of $1.67 \%$ for comparisons on three groups. 


\section{Results}

A number of $42 \mathrm{CM}$ lesions belonging to 39 subjects, 21 female and 18 male (one female subject, 49 years old with two localizations left arm and elbow and one male subject, 75 years old with three localizations - thoracic, scapular and lumbar), mean age of $56.56 \pm 15.44$ years old achieved the inclusion criteria and were assessed in our study.

The main characteristics of the investigated lesions according to the histopathological, Doppler US and SE examinations are presented in Table I.

The Breslow index could not be calculated in 5 lesions (11.9\% [4.8-26.1]), as the histopathological diagnosis was "in situ" and these cases were excluded from further analysis. Finally, 37 lesions were included in the analysis related to the Breslow index.

Overall, no statistical significant difference was observed when the Breslow index was compared with groups of vascularization (low, high, and medium vascularization) $(\mathrm{p}=0.058)$. However, the post-hoc test identified that the mean of the Breslow index on lesions with

Table I. Summary of histopathological, Doppler US and SE examinations

\begin{tabular}{lll}
\hline Characteristics & Value & $\begin{array}{l}\text { Statistics } \\
\text { (p-value) }\end{array}$ \\
\hline $\begin{array}{l}\text { Histology } \\
\text { Breslow index }\end{array}$ & & \\
$\begin{array}{l}\text { Doppler } \boldsymbol{U} \boldsymbol{S} \\
\text { Vascularization }\end{array}$ & & \\
$\quad$ Low vascularization & $26.47[11.85-44.03]$ & $(<0.0001)$ \\
$\quad$ Hypervascularization & $64.71[47.14-79.32]$ & \\
$\quad$ No. of pedicles & & 4.1284 \\
$\quad$ Unique & $29.41[14.79-46.97]$ & $(<0.0001)$ \\
$\quad$ Multiple & $61.76[45.55-78.70]$ & \\
SE & & \\
Elasticity & & $>3.5$ \\
$\quad$ Low & & \\
$\quad$ Medium & $45.24[28.63-61.85]$ & $(<0.0005)^{\mathrm{c}}$ \\
$\quad$ High & $42.86[28.63-59.47]$ & \\
\hline
\end{tabular}

${ }^{\mathrm{a}}$ mean \pm standard deviation; ${ }^{\mathrm{b}}$ percentage $[95 \% \mathrm{CI}]$, where $95 \% \mathrm{CI}=$ $95 \%$ confidence interval; Statistics $=$ the statistics associated to $\mathrm{Z}$ test for proportions; ${ }^{c} \mathrm{Z}$-statistic and associated value on pairs comparisons as low vs. high, respectively medium vs. high

Table II. Doppler US and Breslow index and elastographic appearance respectively: Spearman's correlation coefficients $(\rho)$ and associated significance $(\mathrm{p})$

\begin{tabular}{ll}
\hline Characteristics & $\boldsymbol{\rho}(\mathbf{p}$-value) \\
\hline Vascularization \& the Breslow index & $0.4483(0.0167)$ \\
Number pedicles \& the Breslow index & $0.5016(0.0065)$ \\
SE appearance \& vascularization & $-0.4937(0.0035)$ \\
SE appearance \& the number of pedicles & $-0.5299(0.0015)$ \\
\hline
\end{tabular}

high vascularization was significantly higher compared to the mean of the Breslow index on lesions with low vascularization (high: $3.03 \pm 1.30$; low: $1.57 \pm 0.86$; p-val$\mathrm{ue}=0.018$ ).

The relation between the appearance of vascularization and Breslow index, and the qualitative appearance of the lesion at SE was statistically significant (Table II) and is illustrated in figures 1 and 2 .

The mean value of the Breslow index proved significantly higher whenever multiple pedicles were present compared to cases where there was a unique pedicle (multiple: $3.18 \pm 1.29$, unique: $1.51 \pm 0.71, \mathrm{p}=0.0008$ ).

The lesions with high elasticity proved to have medium or low vascularization while low vascularization was observed in a higher number of cases in lesions with medium elasticity (fig 3). No dependence was observed between the elasticity appearance and vascularization $(p=0.058)$.

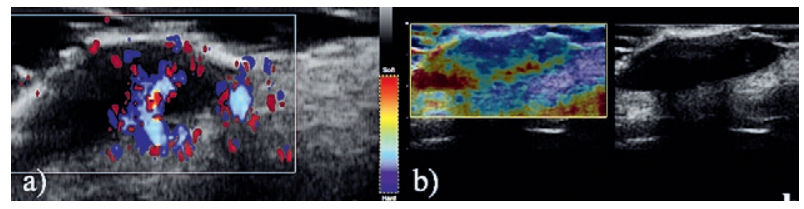

Fig 1. Cutaneous melanoma localized on the right mammary region, hypervascularized on Doppler US (a) and with stiff appearance on SE (b) in a 52 year old female. Breslow index 2.68 $\mathrm{mm}$, thickness of the skin tumor measured by a $40 \mathrm{MHz}$ frequency linear transducer was $2.55 \mathrm{~mm}$.

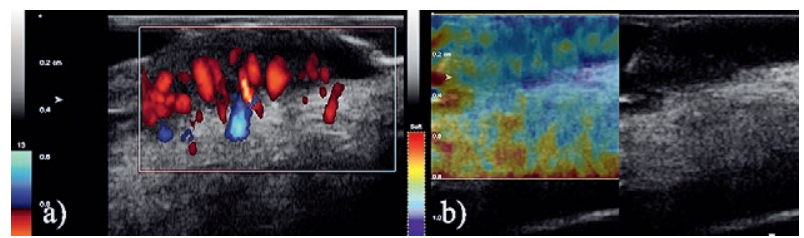

Fig 2. Cutaneous melanoma localized on the left lumbar region with multiple vascular pedicles on Doppler US (a) and stiff appearance on SE (b) in a 57 year old male. Breslow index 3.7 $\mathrm{mm}$, thickness of the skin tumor measured by a $40 \mathrm{MHz}$ frequency linear transducer was $3.8 \mathrm{~mm}$.

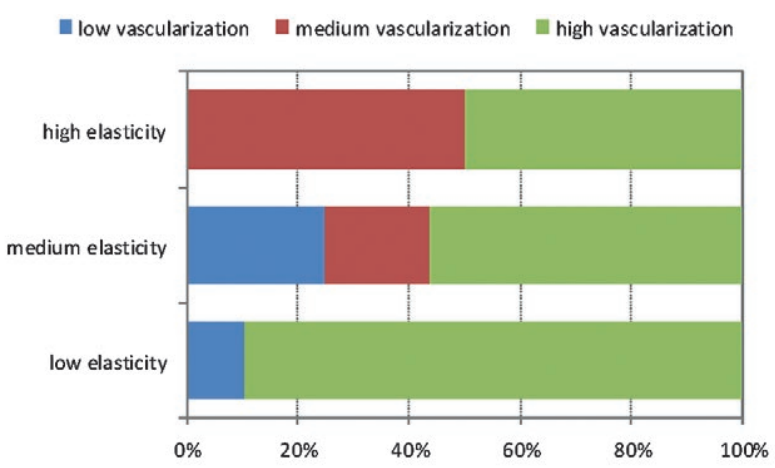

Fig 3. Distribution of SE elasticity according to vascularization 
All lesions with high elasticity had multiple pedicles while the higher percentage of the unique pedicle was observed in lesions with medium elasticity.

No correlation was observed between the elasticity appearance and the number of pedicles $(\mathrm{p}=0.082)$.

\section{Discussions}

To the best of our knowledge this is the first study that compares Doppler US and SE in cutaneous melanoma. It is very important for patients with this pathology to benefit from complex US preoperative diagnosis by assessing not only gray scale appearance, but also vascularization and elasticity.

Doppler US is used to assess the vascularization of the skin tumors before surgical excision, high vascularization being correlated with an important risk of metastasis [17-20]. The thickness of CM lesions represents a very important element for preoperative management and prognosis, being correlated with the risk of metastasis or relapsing, as well $[11,12,21,22]$. Catalano et al [11] found that CMs with a thickness greater than $2 \mathrm{~mm}$ showing high vascularization have a high risk for a bad prognosis. In our study, the median tumor thickness was $2.78 \mathrm{~mm}$, and most of the CMs were very well vascularized. These findings require wide margins of safety at surgery and the exclusion of metastases and, in time, the recurrences. In our group of patients we found that the Breslow index was significantly higher in lesions with high vascularity compared to those with low vascularity; in other words we expected that a lesion with a greater Breslow index would present hypervascularization.

Another investigated and significant factor in our study was the number of vascular pedicles: most tumors presented multiple pedicles $(61.76 \%)$. Di Santolo et al [21] detected an intratumoral vascular signal in most of lesions, with one or two afferent vessels to the lesion. The presence of a greater number of vascular pedicles shows an abundant blood supply. Knowing that vascularization may be related to tumor aggressiveness, through to the metastatic potential or relapsing and reduced longterm survival $[12,19,23,24]$, it is very important for these patients to have a good management both pre- and postoperatively.

The use of elastography in the evaluation of skin lesions has been the subject of several studies, in an attempt to differentiate between benign and malignant lesions [25-27]. Few research studies conducted in the early period of clinical elastography have demonstrated that this technique has potential in the diagnosis of skin tumors. In reported cases the tumors were diagnosed as malignant based on the color distribution on elastographic images [25-27]. In this present study, tumor elasticity represented a statistically significant factor: almost half of the tumors were stiff at SE and another important percentage had medium elasticity. In our patients thicker tumors presented low elasticity, while thin tumors appeared softer. Increased stiffness of melanomas can be explained by increased cellularity and tumor infiltration typical for malignant tumors, which can be correlated with the degree of aggressiveness. Preoperative evaluation of CM by SE is important not only for the tumor itself, but also for perilesional infiltration in order to establish the safety surgical margins.

There are several studies concerning the relationship between the thickness of skin melanomas and the presence and aspect of vascularization. Lassau et al [9] assessed the tumor vascularity using color Doppler in 111 skin melanomas before surgical resection and demonstrated intratumoral vessels in 43 lesions. Lassau et al [9] and Srivastava et al [12] found a strong positive correlation between the vascularization and Breslow tumor thickness, reporting that Breslow thickness and vascularity offer information for the prognosis of these patients. Srivastava et al [12] proposed a "tumor vascularity database" in countries with a high prevalence of melanoma with a detailed description of intratumoral vessels. Catalano et al [11] demonstrated that the presence of intratumoral vessels is correlated with the Breslow index and with patient survival. Di Santolo et al [20] found intratumoral vessels in 24/34 of lesions with thickness greater than $1 \mathrm{~mm}$. We obtained no statistical differences when comparing the Breslow index with the three classes of vascularization but a post hoc test identified that the Breslow index is significantly higher in lesions with hypervascularization compared to those with low vascularity. This confirms that the Breslow index and hypervascularization represents negative prognostic factors for the evolution of patients with CM by increasing the risk of metastasis or relapse $[5,11,21,22]$.

In the present work, we found a strong correlation between the tumor thickness, measured histopathologically by the Breslow index, degree of vascularization, and number of vascular pedicles. We found that as the Breslow index increases more obvious multiple vascular pedicles in the skin tumors can be detected, which demonstrate increased vascularization, consistent with a previous published study [9]. To the best of our knowledge, the relation between the elastographic appearance of $\mathrm{CM}$ and the aspect of vascularization and number of vascular pedicles has not been previously described. We noticed that the SE appearance of skin 
melanoma is strongly correlated both with the vascularization and with the number of vascular pedicles of the lesions. We found that tumors with high elasticity presented medium or low vascularization. This fact could be correlated with the aggressiveness of the tumor, assuming that an increased vascularity is associated with an increased degree of aggressiveness, and a malignant tumor tissue shows increased stiffness. In our study we could not find a model of correlation between the SE appearance of CM and their degree of vascularization and the number of vascular pedicles, probably due to the variability of the tumor thickness.

Since it has been proven that hypervascularization is a bad prognostic factor $[12,20]$ and we have proved that there is a positive correlation between the stiffness and degree of vascularization, our results suggest that the stiffness of the tumor represents a negative prognostic factor. However, it is necessary to analyze a larger group of patients and their clinical outcome to assess the extent to which the stiffness is an independent predictor of unfavorable evolution.

One limitation of the study arises from the small number of melanoma tumors enrolled. This item represents only a part of the study, which is still ongoing. Another limitation of our work can be the bias induced by the fact that the Doppler and SE examinations were performed by the same investigator and no inter- and intra-observer could be computed. A greater degree of objectivity could be obtained by measuring the Doppler indices. Another limitation resides in the qualitative assessment of elasticity by means of SE scores. Shear wave elastography might represent another, more objective, way of quantifying stiffness, but this method was not available for this study.

To the best of our knowledge, data on the correlation between the intratumoral vascular signal and the elastographic appearance has not been previously published. Further studies on larger groups of patients are necessary to confirm and refine the results of the present work.

\section{Conclusions}

This study demonstrates that the assessment of intratumoral vascularization and stiffness in skin melanomas may contribute to a complex diagnosis before surgical excision. Both Doppler US and SE may provide useful information for the management of CM patients. We recommend the association of these techniques, whenever possible, with a two dimensional US and clinical examination for a complex and complete diagnosis of cutaneous melanoma.

\section{Acknowledgements}

The study was supported by the "Iuliu Haţieganu" University of Medicine and Pharmacy, Cluj-Napoca through project no. 1494/7/28.01.2014.

\section{Conflict of interest: none}

\section{References}

1. Tronnier M, Semkova K, Wollina U, Tchernev G. Malignant melanoma: epidemiologic aspects, diagnostic and therapeutic approach. Wien Med Wochenschr 2013; 163: 354-358.

2. Voit CA, Gooskens SL, Siegel P, et al. Ultrasound-guided fine needle aspiration cytology as an addendum to sentinel lymph node biopsy can perfect the staging strategy in melanoma patients. Eur J Cancer 2014; 50: 2280-2288.

3. Livingstone E, Krajewski C, Eigentler TK, et al. Prospective evaluation of follow-up in melanoma patients in Germany - Results of a multicentre and longitudinal study. Eur J Cancer 2015; 51: 653-667.

4. Scally CP, Wong SL. Intensity of follow-up after melanoma surgery. Ann Surg Oncol 2014; 21: 752-757.

5. Li J, Wang Y, Liang R, et al. Recent advances in targeted nanoparticles drug delivery to melanoma. Nanomedicine 2015; 11: 769-794.

6. Scolyer RA, Prieto VG. Melanoma pathology: important issues for clinicians involved in the multidisciplinary care of melanoma patients. Surg Oncol Clin N Am 2011; 20: $19-37$.

7. Patnana M, Bronstein Y, Szklaruk J, et al. Multimethod imaging, staging, and spectrum of manifestations of metastatic melanoma. Clin Radiol 2011; 66: 224-236.

8. Srivastava A, Hughes LE, Woodcock JP, Laidler P. Vascularity in cutaneous melanoma detected by Doppler sonography and histology: correlation with tumor behaviour. Br J Cancer 1989; 59: 89-91.

9. Lassau N, Lamuraglia M, Koscielny S, et al. Prognostic value of angiogenesis evaluated with high-frequency and colour Doppler sonography for preoperative assessment of primary cutaneous melanomas: correlation with recurrence after a 5 year follow-up period. Cancer Imaging 2006; 6: 24-29.

10. Dancey AL, Mahon BS, Rayatt SS. A review of diagnostic imaging in melanoma. J Plast Reconstr Aesthet Surg 2008; 61: 1275-1283.

11. Catalano O, Siani A. Cutaneous melanoma: role of ultrasound in the assessment of locoregional spread. Curr Probl Diagn Radiol 2010; 39: 30-36.

12. Srivastava A, Woodcock JP, Mansel RE, et al. Doppler ultrasound flowmetry predicts 15 year outcome in patients with skin melanoma. Indian J Surg 2012; 74: 278-283.

13. Hernández C, del Boz J, de Troya M. can high-frequency skin ultrasound be used for the diagnosis and management of basal cell carcinoma? Actas Dermosifiliogr 2014; 105: 107-111. 
14. Botar-Jid C, Vasilescu D, Dudea SM. Tridimensional ultrasound and Sonoelastography in musculoskeletal pathology. In: Fodor D, Clinical musculoskeletal ultrasound. Bucureşti, Ro: Medical Ed, 2009: 381-389.

15. Bamber J, Cosgrove D, Dietrich CF, et al. EFSUMB guidelines and recommendations on the clinical use of ultrasound elastography. Part 1: Basic principles and technology. U1traschall Med 2013; 34: 169-184.

16. Jäntschi L, Bolboacă SD. Exact probabilities and confidence limits for binomial samples: applied to the difference between two proportions. ScientificWorldJournal 2010; 10: 865-878.

17. Doben AR, MacGillivray DC. Current concepts in cutaneous melanoma: malignant melanoma. Surg Clin North Am 2009; 89: 713-725.

18. Leiter U, Buettner PG, Eigentler TK, et al. Hazard rates for recurrent and secondary cutaneous melanoma: An analysis of 33,384 patients in the German Central Malignant Melanoma Registry. J Am Acad Dermatol 2012; 66: 37-45.

19. Mandava A, Ravuri PR, Konathan R. High-resolution ultrasound imaging of cutaneous lesions. Indian J Radiol Imaging 2013; 23: 269-277.

20. Scotto di Santolo M, Sagnelli M, Mancini M, et al. Highresolution color-Doppler ultrasound for the study of skin growths. Arch Dermatol Res 2015; 307: 559-566.
21. Wortsman X. Common applications of dermatologic sonography. J Ultrasound Med 2012; 31: 97-111.

22. Kleinerman R, Whang TB, Bard RL, Marmur ES. Review ultrasound in dermatology: principles and applications. J Am Acad Dermatol 2012; 67: 478-487.

23. Wortsman X, Wortsman J. Clinical usefulness of variablefrequency ultrasound in localized lesions of the skin. J Am Acad Dermatol 2010; 247-256.

24. Alfageme Roldán F. Ultrasound skin imaging. Actas Dermosifiliogr 2014; 105: 891-899.

25. Hinz T, Wenzel J, Schmid-Wendtner MH. Real-time tissue elastography: A helpful tool in the diagnosis of cutaneous melanoma? J Am Acad Dermatol 2011; 65: 424-426.

26. Hinz T, Hoeller T, Wenzel J, Bieber T, Schmid-Wendtner MH. Real-time tissue elastography as promising diagnostic tool for diagnosis of lymph node metastases in patients with malignant melanoma: a prospective single-center experience. Dermatology 2013; 226: 81-90.

27. Wortsman X, Carreńo L, Morales C. Skin Cancer: The Primary Tumors. In: Wortsman X, Jemec GBE, eds. Dermatologic Ultrasound with Clinical and Histologic Correlations. New York, USA: Springer, 2013: 249-280. 